\title{
Effect of amorphous phase separation and crystallization on the in vitro and in vivo performance of an amorphous solid dispersion
}

Knopp, Matthias Manne; Wendelboe, Johan; Holm, René; Rades, Thomas

Published in:

European Journal of Pharmaceutics and Biopharmaceutics

DOI:

10.1016/j.ejpb.2018.07.005

Publication date:

2018

Document Version

Peer reviewed version

Citation for published version (APA):

Knopp, M. M., Wendelboe, J., Holm, R., \& Rades, T. (2018). Effect of amorphous phase separation and crystallization on the in vitro and in vivo performance of an amorphous solid dispersion. European Journal of Pharmaceutics and Biopharmaceutics, 130, 290-295. https://doi.org/10.1016/j.ejpb.2018.07.005

\section{General rights}

Copyright and moral rights for the publications made accessible in the public portal are retained by the authors and/or other copyright owners and it is a condition of accessing publications that users recognise and abide by the legal requirements associated with these rights.

- Users may download and print one copy of any publication from the public portal for the purpose of private study or research.

- You may not further distribute the material or use it for any profit-making activity or commercial gain.

- You may freely distribute the URL identifying the publication in the public portal.

Take down policy

If you believe that this document breaches copyright please contact rucforsk@kb.dk providing details, and we will remove access to the work immediately and investigate your claim. 


\section{Accepted Manuscript}

Effect of amorphous phase separation and crystallization on the in vitro and in vivo performance of an amorphous solid dispersion

Matthias Manne Knopp, Johan Wendelboe, René Holm, Thomas Rades

PII:

$$
\text { S0939-6411(18)30694-5 }
$$

DOI: https://doi.org/10.1016/j.ejpb.2018.07.005

Reference:

EJPB 12828

To appear in:

European Journal of Pharmaceutics and Biophar-

maceutics

Received Date: $\quad 31$ May 2018

Revised Date: $\quad 2$ July 2018

Accepted Date: $\quad 3$ July 2018

Please cite this article as: M. Manne Knopp, J. Wendelboe, R. Holm, T. Rades, Effect of amorphous phase separation and crystallization on the in vitro and in vivo performance of an amorphous solid dispersion, European Journal of Pharmaceutics and Biopharmaceutics (2018), doi: https://doi.org/10.1016/j.ejpb.2018.07.005

This is a PDF file of an unedited manuscript that has been accepted for publication. As a service to our customers we are providing this early version of the manuscript. The manuscript will undergo copyediting, typesetting, and review of the resulting proof before it is published in its final form. Please note that during the production process errors may be discovered which could affect the content, and all legal disclaimers that apply to the journal pertain. 


\section{Effect of amorphous phase separation and crystallization on}

\section{the in vitro and in vivo performance of an amorphous solid dispersion}

Matthias Manne Knopp, ${ }^{1}$ Johan Wendelboe, ${ }^{2,3}$ René Holm, ${ }^{3,4 *}$ Thomas Rades, ${ }^{2}$

${ }^{1}$ Bioneer:FARMA, Department of Pharmacy, DK-2100 Copenhagen, Denmark

${ }^{2}$ Department of Pharmacy, University of Copenhagen, DK-2100 Copenhagen, Denmark

${ }^{3}$ Drug Product Development, Janssen Research and Development, Johnson \& Johnson, BE-2430

Beerse, Belgium

${ }^{4}$ Department of Science and Environment, Roskilde University, DK-4000 Roskilde, Denmark

"Correspondence to: René Holm, Janssen Pharmaceutica, Johnson and Johnson, Turnhoutseweg 30, BE-2340 Beerse, Belgium (Telephone: +32-01460-8107; Fax: +32 01460-2841; E-mail: rholm@its.jnj.com)

Keywords

Amorphous solid dispersion; bioavailability; phase separation; crystallization; IVIVC 


\section{Abstract}

2 The purpose of this study was to investigate the influence of amorphous phase separation and

3 erystallization of the drug in an amorphous solid dispersion (ASD) on its in vitro and in vivo

4 performance. The amorphous phase-separated or crystallized ASDs were prepared by mixing a

5 homogenous ASD (glass solution) of the model drug celecoxib (CCX) in polyvinylpyrrolidone

6 (PVP) with pure amorphous or micronized crystalline CCX at 20, 40, 60 or 100\% of the total

7 drug load (25:75 w/w CCX:PVP), respectively. In this study, the performance of phase separated

8 and crystallized amorphous solid dispersions (ASDs) these formulations-was then-evaluated by

9 non-sink in vitro dissolution testing in fasted-state simulated intestinal fluid (FaSSIF) and in vivo

10 in rats and compared to that of crystalline CCX, amorphous CCX and pure ASD. The amorphous

11 phase-separated or crystallized ASDs were prepared by mixing an ASD of the model drug

12 celecoxib (CCX) in polyvinylpyrrolidone (PVP) with pure amorphous or micronized crystalline

$13 \mathrm{CCX}$ at 20,40,60 or $100 \%$ of the total drug load (25:75 w/w CCX:PVP), respectively. As

14 expected, crystallization of CCX in the ASDs generally had a negative influence on both the area

15 under the curve of the dissolution curve (in vitro AUC) and the plasma concentration-time

16 profile (in vivo AUC) in rats compared to the pure ASD. However, the difference between the in

17 vivo AUC of the pure ASD and the $20 \%$ and $40 \%$ crystallized ASDs was not statistically

18 significant, which could indicate that a low fraction of crystallization of a drug in an ASD may

19 only have limited impact on in vivo performance and hence bioavailability. In comparison,

20 amorphous phase separation of CCX in the ASDs did not negatively influence the in vitro AUC

21 and in vivo AUC to the same degree as crystallization and the dissolution profiles of all the

22 amorphous phase-separated ASDs were similar to that of the pure ASD. In fact, even though a

23 slight decrease of in vivo AUC with increasing fraction of amorphous phase separation was

24 observed, the $20 \%$ and $40 \%$ amorphous phase-separated ASDs were bioequivalent with the pure

25 ASD. Consequently, even though only one drug polymer systems was investigated, the findings

26 of this study indicate that the current regulatory general reservations towards the impact of

27 amorphous phase separation and crystallization of the drug in an ASD on its bioavailability may

28 be exaggerated. 


\section{Introduction}

Oral drug delivery has always been the preferred route of administration of pharmaceuticals, both from the patient's perspective due to convenience and compliance, and from the manufacturer's perspective due to long shelf life and limited cost of production (Augustijns and Brewster, 2012; Leuner and Dressman, 2000; Baghel et al., 2016). However, as a result of advanced high-throughput screening methods and the implementation of computational chemistry, the pharmaceutical industry is currently experiencing an increase in the amount of complex drug molecules. These more complex molecules in most cases entail a solubility limitation that challenges the oral delivery of these drugs, and has prompted the development of different so-called enabling formulation strategies to overcome the solubility limitations, including amorphous solid dispersions (ASDs) (Van den Mooter, 2012; Baghel et al., 2016; Stegemann et al., 2007; Fahr and Liu, 2007). An ASD is a molecular dispersion of a drug in an amorphous polymer, commonly prepared by melt-based method such as hot-melt extrusion or solvent-based method such as spray drying, and less commonly by freeze-drying or milling (Vo et al., 2013; Brouwers et al., 2009; Leuner and Dressman, 2000). The aim of this enabling formulation approach is first of all to increase the free energy of the drug and thus, its apparent solubility through amorphization and secondly, to stabilize the inherently unstable amorphous form of the drug through intermolecular interactions with the polymer (Van den Mooter, 2012; Warren et al, 2010). Compared to pure amorphous drugs, ASDs have a higher physical and chemical stability due to reduced molecular mobility, which also will increase the glass transition temperature of the system (Hancock and Zografi, 1997).

To reduce the "pill burden" and increase compliance, many of the currently marketed ASDs are formulated with a relatively high drug loading. However, even though it is physically stabilized in a polymer, the drug in an ASD can also be thermodynamically unstable if the drug load exceeds its equilibrium solubility in the polymer at storage temperature (Marsac et al., 2009; Knopp et al., 2015). This situation will eventually lead to two potential outcomes: either the drug will separate to form local amorphous drug-rich clusters (amorphous phase separation) and/or the drug will nucleate and crystallize. Both of these events will significantly change the physicochemical properties of the formulation (Newman et al, 2012; Baghel et al., 2016). Consequently, overloading the drug in the ASD will not only affect the overall physical stability, 
but may also lead to variable performance and ultimately lower bioavailability over time and hence, constitutes a major regulatory, efficiency and safety issue (Rahman et al., 2014).

Therefore, the aim of this study was to evaluate the influence of amorphous phase separation and crystallization on the in vitro and in vivo performance of an ASD of celecoxib (CCX) in polyvinylpyrrolidone (PVP). The amorphous phase-separated and crystallized ASDs were prepared by mixing a homogenous ASD (glass solution) of CCX in PVP with pure amorphous or micronized crystalline CCX at 20, 40, 60 or $100 \%$ of the total drug load $(25: 75 \mathrm{w} / \mathrm{w}$ CCX), respectively. The performance of these formulations was then evaluated by non-sink in vitro dissolution testing in fasted-state simulated intestinal fluid (FaSSIF) and in vivo in rats and compared to that of crystalline CCX, amorphous CCX and pure ASD.

\section{Materials and methods}

\section{Materials}

Celecoxib $\left(\mathrm{CCX}, M_{\mathrm{w}}=381.37 \mathrm{~g} / \mathrm{mol}\right)$, sodium chloride, sodium dihydrogen phosphate monohydrate, sodium hydroxide, hydrogen chloride, $0.1 \%$ trifluoracetic acid and acetonitrile were purchased from VWR (Leuven, Belgium). Kollidon ${ }^{\circledR} 30$ (PVP, $M_{\mathrm{w}}=44,000-54,000$ g/mol) was kindly supplied by BASF (Ludwigshafen, Germany) and SIFTM Powder instant biorelevant medium was purchased from Biorelevant.com Ltd (London, United Kingdom).

\section{Sample preparation}

The pure ASD and amorphous CCX were prepared by melt quenching. CCX and PVP were weighed (25\% w/w CCX) and mixed thoroughly using a mortar and pestle. The physical mixture was then spread evenly on aluminum foil and placed in an electrical furnace at $169{ }^{\circ} \mathrm{C}$ for 5 min. The mixture was removed from the furnace, allowed to cool to room temperature and pulverized using a mortar and pestle. For the ASD this procedure was repeated to ensure homogeneity. The resulting powders were sieved through a $0.2 \mathrm{~mm}$ sieve and stored in air-tight amber containers until use.

For the preparation of the amorphous phase-separated and crystallized ASDs, ASDs of different CCX concentration $(5,10,15$ and $25 \% \mathrm{w} / \mathrm{w})$ were prepared using the aforementioned melt quenching method and mixed thoroughly with micronized pre-milled crystalline CCX (to better mimic the particle size of crystallized drug in the ASD) or freshly prepared amorphous CCX 
using a mortar and pestle to yield 20,40, 60 or $100 \%$ amorphous phase-separated or crystallized ASD. In all the ASDs, the total CCX concentration was fixed at $25 \% \mathrm{w} / \mathrm{w}$ and named after their relative fraction of amorphous phase-separated or crystallized CCX. For example, 40\% amorphous phase-separated ASD refers to a system where $40 \%$ of the total dose of CCX has amorphous phase-separated and $60 \%$ is still dispersed/dissolved in the polymer. The successful formation of amorphous phase-separated ASDs (as evident from two separate glass transitions and no melting event) and crystallized ASDs (as evident from a single glass transition and a melting event) was confirmed using differential scanning calorimetry and X-ray powder diffraction (see Supplementary Material) (data now shown).

\section{Preparation of dissolution media}

For the non-sink dissolution studies, FaSSIF (version 1) was used as the dissolution medium and was prepared following the instructions provided by Biorelevant.com. To prepare $1 \mathrm{~L}$ of FaSSIF, $1 \mathrm{~L}$ of pH $6.520 \mathrm{mM}$ phosphate buffer was first prepared by dissolving $0.42 \mathrm{~g}$ sodium hydroxide pellets, $3.95 \mathrm{~g}$ sodium dihydrogen phosphate monohydrate and $6.19 \mathrm{~g}$ of sodium chloride in 900 $\mathrm{mL}$ of purified filtered Milli-Q water. The $\mathrm{pH}$ was then adjusted to $6.50 \pm 0.05$ with $0.1 \mathrm{~N}$ sodium hydroxide or hydrochloric acid and finally, the volume was increased to $1000 \mathrm{~mL}$ with Milli-Q water. A total of $2.12 \mathrm{~g}$ of the SIF powder was weighed and added to approximately $500 \mathrm{~mL}$ of the phosphate buffer and when completely dissolved, the volume was increased to $1000 \mathrm{~mL}$ with the buffer and kept on a magnetic stirrer for at least $2 \mathrm{~h}$ until use. The FaSSIF was used within 24 $\mathrm{h}$ of preparation as recommended by the manufacturer.

\section{In vitro dissolution}

The non-sink dissolution studies were performed on a VanKel VK 7000 USP II apparatus equipped with a VK 750D/K heating device from VanKel Technology Group (Cary, NC, USA). A total of $300 \mathrm{~mL}$ of freshly prepared FaSSIF was added to each dissolution chamber and allowed to equilibrate at $37^{\circ} \mathrm{C}$. The remaining FaSSIF was stored in an oven at $37.5{ }^{\circ} \mathrm{C}$. The dissolution studies were initialized by pre-suspending the formulation at a dose drug load of corresponding to $400 \mathrm{mg}$ CCX in $100 \mathrm{~mL} \mathrm{FaSSIF}$ in a $150 \mathrm{~mL}$-beaker-under by rigorous stirring. This suspension was then immediately added to the dissolution chamber, and an additional 100 $\mathrm{mL} \mathrm{FaSSIF}$ was used to rinse the beaker of any remaining drug formulation and added to the 
dissolution chamber to yield a total of $500 \mathrm{~mL} \mathrm{FaSSIF} \mathrm{(and} 800 \mathrm{~m} \mu \mathrm{g} / \mathrm{mL}$ CCX). Samples of 2 $\mathrm{mL}$ were withdrawn from each chamber at predetermined time points $(1,5,10,15,20,30,45$, $60,90,120,180,240,360$ and $1440 \mathrm{~min}$ ) with a syringe and filtered into a $4 \mathrm{~mL}$ black cap vial with a Spartan Whatman $0.22 \mu \mathrm{m}$ syringe filter from GE Health Care (Chicago, IL, USA). From this vial, $1 \mathrm{~mL}$ of the filtrate was immediately diluted with $1 \mathrm{~mL}$ of methanol to prevent precipitation from supersaturated samples and assayed for CCX content by UPLC (see below).

\section{In vivo study}

The procedure for the in vivo study was approved by the local Animal Welfare Committee. All animal procedures were carried out in compliance with EC Directive 2010/63/ EU, NIH Guide for the Care and Use of Laboratory Animals, and with the Belgian laws regulating experiments on animals.

Male Sprague-Dawley rats ( 300 g) were purchased from Charles River Laboratories (Sulzfeld, Germany). To avoid food effects, all rats were fasted 16-20 h prior to the study and until $8 \mathrm{~h}$ after dosing. Immediately before oral dosing, the formulations were suspended in $30 \mathrm{~mL} \mathrm{FaSSIF}$ at a concentration corresponding to $10 \mathrm{mg} / \mathrm{mL}$ CCX using a magnetic stirrer and dosed to each rat individually at $10 \mathrm{~mL} / \mathrm{kg}$ by oral gavage $(n=3-5)$. A total of 42 rats were randomly assigned to one of the following groups, each consisting of 3-5 animals: i) amorphous $\operatorname{CCX}(n=5)$, ii) pure ASD $(25: 75 \mathrm{w} / \mathrm{w})$ of CCX-PVP $(n=5)$, iii) $20 \%$ crystallized ASD $(n=5)$, iv) $40 \%$ crystallized ASD $(n=5), v) 60 \%$ crystallized ASD $(n=5)$, vi) $100 \%$ crystallized ASD $(n=5)$, vii), 20\% amorphous phase-separated $\operatorname{ASD}(n=3)$, viii) $40 \%$ amorphous phase-separated ASD $(n=3)$, ix) $60 \%$ amorphous phase-separated $\operatorname{ASD}(n=3), \mathrm{x}) 100 \%$ amorphous phase-separated ASD $(n=3)$. Blood samples of $100 \mu \mathrm{L}$ were collected at $0.5,1,2,3,4,6,8$ and $24 \mathrm{~h}$ after administration by individual lateral tail vein puncture and transferred to ethylenediaminetetraacetic acid (EDTA)-coated tubes to prevent coagulation. The blood samples were then centrifuged for $10 \mathrm{~min}$ at $3600 \times \mathrm{g}$ and the plasma was subsequently transferred to labeled plastic tubes and stored at $-80^{\circ} \mathrm{C}$ until analysis. After collection of the last sample, the animals were euthanized.

\section{Quantitative analysis}


The method of analysis for the in vitro dissolution samples was developed on an Acquity ultra performance liquid chromatograph (UPLC) equipped with an Acquity UPLC BEH C18 column, both from Waters Corp. (Milford, MA, USA), using a mobile phase consisting of triflouroacetic acid (TFA) $0.1 \%$ in water and acetonitrile in a 70:30 v/v ratio, respectively. The diluted samples were mixed on a VVR whirl-mixer and added to a 96 well plate in which $100 \mu \mathrm{g} / \mathrm{mL}$ CCX in methanol standards were added as a reference. A volume of 1-3 $\mu \mathrm{L}$ was eluted at a flow rate of $0.6 \mathrm{ml} / \mathrm{min}$ with a column oven temperature of $55{ }^{\circ} \mathrm{C}$, and the effluent was detected at a wavelength of $251 \mathrm{~nm}$ after approximately 1.7 minutes. A standard curve was prepared by analyzing CCX in methanol in concentrations between $1 \mu \mathrm{g} / \mathrm{mL}$ and $250 \mu \mathrm{g} / \mathrm{mL}$, resulting in a standard curve with a linear correlation of $\mathrm{R}^{2}=0.999$.

The concentration of CCX in plasma was determined using UPLC chromatography with MS/MS detection. A quantity of $10 \mu \mathrm{L}$ plasma was mixed with $10 \mu \mathrm{L}$ blank plasma, $20 \mu \mathrm{L}$ DMSO and $200 \mu \mathrm{L}$ of acetonitrile. The samples were centrifuged at $6,000 \times \mathrm{g}$ for $20 \mathrm{~min}$ at $5^{\circ} \mathrm{C}$. Analysis of the plasma samples was performed by UPLC connected to a tandem mass spectrometer (MS/MS) using a Waters Acquity Ultra Performance Liquid Chromatograph system (Waters Corp., Milford, MA) equipped with a binary solvent delivery system (pump), a sample manager module with autosampler, and a column compartment/heater. A switch valve connected the UPLC to the mass spectrometer. The MS/MS detection was performed using a SCIEX API 4000 MS/MS system with a Turbo Ion Spray® (ESI) as an interface (Applied Biosystems, Carlsbad, CA), operating in the positive ion electrospray mode. Operational settings: CCX was detected at a precursor product ion mass to charge ratio $(\mathrm{m} / \mathrm{z})$ of 316 . Collision gas $(\mathrm{CAD})$ 6.0, temperature (TEM) $550{ }^{\circ} \mathrm{C}$, IS $-4500 \mathrm{~V}$, Entrance potential (EP) - 10.0 V and collision energy (CE) - 32.0 V. For the UPLC analysis, chromatographic separation was performed on a Acquity UPLC BEH C18 $(50 \times 2.1 \mathrm{~mm}, 1.7 \mu \mathrm{m})$ from Waters Corp. (Milford, MA, USA). The mobile phases contained $0.1 \%$ formic acid in water (A) and methanol (B). The total run time was 1.7 min and a gradient system was used. The gradient conditions were $80.0 \% \mathrm{~A}$ and $20.0 \% \mathrm{~B}$ from initial time to $1 \mathrm{~min}, 90 \% \mathrm{~A}$ and $10 \% \mathrm{~B}$ from 1 to $1.15 \mathrm{~min}$ and finally $80.0 \% \mathrm{~A}$ and $20.0 \% \mathrm{~B}$ from 1.15 to $1.7 \mathrm{~min}$. The flow rate was $0.80 \mathrm{~mL} / \mathrm{min}$. The column temperature was maintained at $40{ }^{\circ} \mathrm{C}$, and the injection volume was $1.0 \mu \mathrm{L}$. A lower limit of quantification (LLOQ) and an upper limit of quantification (ULOQ) of 10 and $40000 \mathrm{ng} / \mathrm{mL}$, respectively, were obtained. 


\section{Data analysis}

Results from the in vitro study are expressed as mean $\pm \mathrm{SD}$ and data from the in vivo study are expressed as mean \pm SEM. Statistical analysis of the in vivo data was performed in SigmaPlot 13.0 from Systat Software Inc. (Chicago, IL, USA). The primary pharmacokinetic parameters: area under the plasma concentration-time profile $\left(\mathrm{AUC}_{0-24 \mathrm{~h}}\right)$, maximum plasma concentration $\left(C_{\max }\right)$ and time to reach $C_{\max }\left(t_{\max }\right)$ were obtained by non-compartmental analysis of the plasma data. A one-way analysis of variance (ANOVA) followed by a pairwise multiple comparison Student-Newman-Keuls test was performed on untransformed data in order to identify significant differences of $C_{\max }$ and $\mathrm{AUC}_{0-24 \mathrm{~h}}$ and differences in $t_{\max }$ were analyzed using a MannWhitney rank sum test for the paired samples. A statistical $p$ value $<0.05$ was considered significant.

\section{Results and discussion}

\section{Crystallization}

The in vitro dissolution profiles and in vivo plasma concentration-time profiles following administration of the different crystallized ASDs are shown in Figures 1 and 2, respectively. The corresponding (pharmacokinetic) parameters, maximum (plasma) concentration $\left(C_{\max }\right)$ of $\mathrm{CCX}$, time to reach $C_{\max }\left(t_{\max }\right)$, and area under the dissolution $\left(\mathrm{AUC}_{0-4 \mathrm{~h}}\right)$ and plasma concentration-time curves $\left(\mathrm{AUC}_{0-24 \mathrm{~h}}\right)$ are provided in Tables 1 and 2, respectively. The area under the dissolution curve (in vitro $\mathrm{AUC}_{0-4 h}$ ) is calculated from $0-4$ hours as the gastrointestinal transit time of rats is 3-4 hours and a previous study showed that this concentration-time profile is predictive of in vivo bioavailability of CCX ASDs (Knopp et al., 2016). 


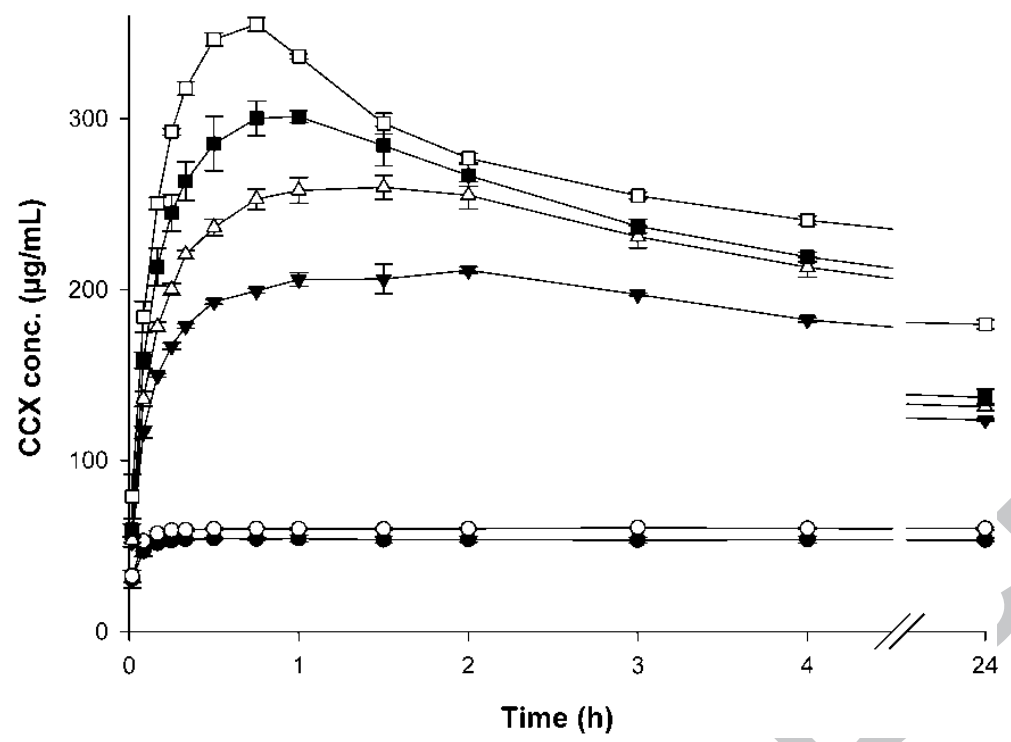

Figure 1: Dissolution profiles after non-sink in vitro dissolution of crystalline CCX $(\bullet), 100 \%$ crystallized ASD (०), 60\% crystallized ASD ( $\nabla), 40 \%$ crystallized ASD $(\triangle), 20 \%$ crystallized ASD ( $\square$ ) and pure ASD ( $\square$ ) at a dose of $400 \mathrm{mg}$ of CCX in $500 \mathrm{~mL}$ FaSSIF. Values represent mean CCX concentration $\pm \mathrm{SD}(n=3)$.

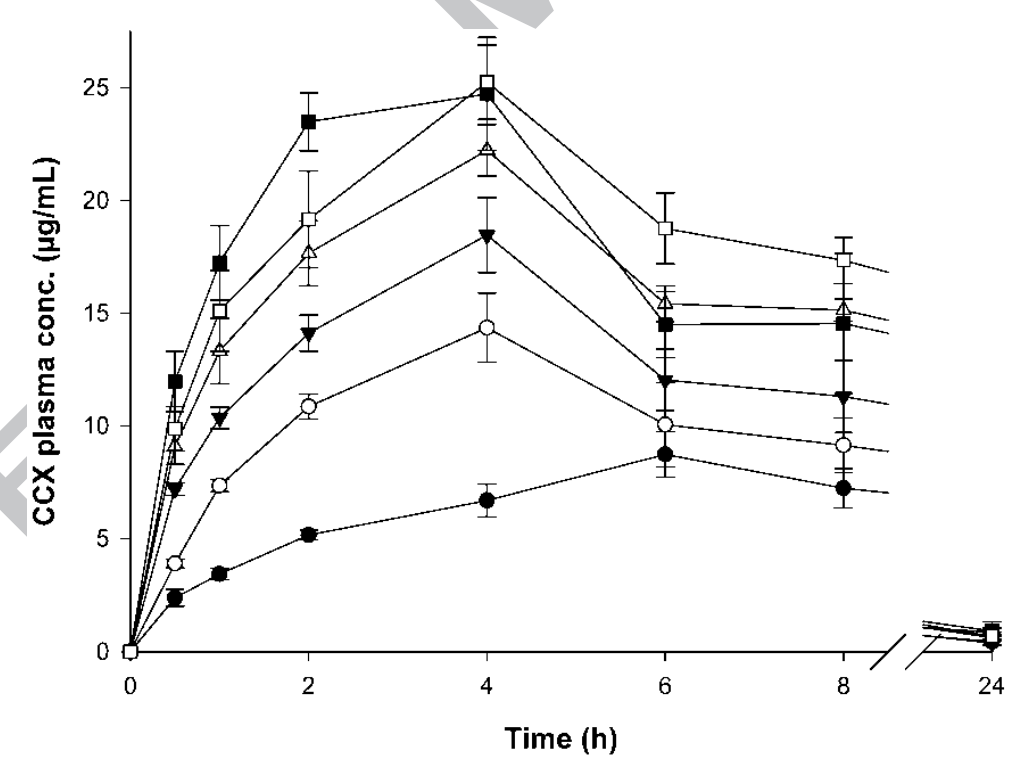

Figure 2: Plasma concentration-time profiles after oral administrations of crystalline CCX (•), $100 \%$ crystallized ASD (०), 60\% crystallized ASD ( $\mathbf{\nabla}), 40 \%$ crystallized ASD $(\triangle), 20 \%$ crystallized ASD ( $\square$ ) and pure ASD ( $\square$ ) in rats at a dose corresponding to $100 \mathrm{mg} / \mathrm{kg}$ body weight of CCX. Values represent mean plasma concentration $\pm \operatorname{SEM}(n=5)$. 
Table 1: In vitro parameters after non-sink dissolution testing of the crystallized ASDs at a dose of $400 \mathrm{mg}$ (values are mean $\pm \mathrm{SD}, n=3$ ).

\begin{tabular}{|l|l|l|l|}
\hline Formulation & $\mathbf{A U C}_{\mathbf{0 - 4}}\left(\mathbf{m g ~} \mathbf{~ m i n} \mathbf{~ m L}^{-\mathbf{1}}\right)$ & $\boldsymbol{C}_{\max }\left(\boldsymbol{\mu g} \mathbf{~ m L}^{-\mathbf{1}}\right)$ & $\boldsymbol{t}_{\max }(\mathbf{m i n})$ \\
\hline Pure ASD & $67.2 \pm 0.3$ & $355.2 \pm 4.0$ & $45.0 \pm 0.0$ \\
\hline $\mathbf{2 0 \%}$ crystallized ASD & $61.3 \pm 0.5$ & $303.1 \pm 5.0$ & $50.0 \pm 8.7$ \\
\hline $\mathbf{4 0 \%}$ crystallized ASD & $56.4 \pm 1.5$ & $260.0 \pm 7.1$ & $80.0 \pm 17.3$ \\
\hline $\mathbf{6 0 \%}$ crystallized ASD & $46.6 \pm 0.3$ & $212.4 \pm 3.4$ & $110.0 \pm 17.3$ \\
\hline $\begin{array}{l}\mathbf{1 0 0 \%} \text { crystallized } \\
\text { ASD }\end{array}$ & $14.3 \pm 0.0$ & $60.8 \pm 0.2$ & $\mathrm{a}$ \\
\hline Crystalline CCX & $12.8 \pm 0.3$ & $54.9 \pm 1.4$ & $\mathrm{a}$ \\
\hline
\end{tabular}

${ }^{\mathrm{a}} t_{\max }$ at end of sampling period (1440 min)

Table 2: Pharmacokinetic parameters of the various crystallized ASDs administered to rats at a dose of $100 \mathrm{mg} / \mathrm{kg}$ (values are mean \pm SEM, $n=5$ ).

\begin{tabular}{|l|l|l|l|}
\hline Formulation & $\mathbf{A U C}_{\mathbf{0 - 2 4}} \mathbf{~}\left(\boldsymbol{\mu} \mathbf{~ h ~} \mathbf{~ m L}^{-\mathbf{1}}\right)$ & $\boldsymbol{C}_{\max }\left(\boldsymbol{\mu} \mathbf{g} \mathbf{~ m L}^{-\mathbf{1}}\right)$ & $\boldsymbol{t}_{\max }(\mathbf{h})$ \\
\hline Pure ASD & $294.4 \pm 16.4^{\mathrm{a}, \mathrm{b}, \mathrm{c}}$ & $25.7 \pm 1.4^{\mathrm{a}, \mathrm{b}, \mathrm{c}}$ & $3.6 \pm 0.4^{\mathrm{a}}$ \\
\hline $\mathbf{2 0 \%}$ crystallized ASD & $270.8 \pm 39.4^{\mathrm{a}, \mathrm{b}}$ & $25.4 \pm 2.2^{\mathrm{a}, \mathrm{b}, \mathrm{c}}$ & $3.2 \pm 0.4^{\mathrm{a}}$ \\
\hline $\mathbf{4 0 \%}$ crystallized ASD & $257.5 \pm 9.0^{\mathrm{a}, \mathrm{b}}$ & & $4.0 \pm 0.0^{\mathrm{a}}$ \\
\hline $\mathbf{6 0 \%}$ crystallized ASD & $198.3 \pm 21.3^{\mathrm{a}}$ & $22.2 \pm 1.1^{\mathrm{a}, \mathrm{b}}$ & $4.0 \pm 0.0^{\mathrm{a}}$ \\
\hline $\begin{array}{l}\mathbf{1 0 0 \%} \text { crystallized } \\
\text { ASD }\end{array}$ & $158.3 \pm 17.8$ & $18.5 \pm 1.7^{\mathrm{a}}$ & $4.0 \pm 0.0^{\mathrm{a}}$ \\
\hline \begin{tabular}{l} 
Crystalline CCX \\
\hline
\end{tabular} & $105.1 \pm 10.2$ & $14.4 \pm 1.5^{\mathrm{a}}$ & $6.3 \pm 0.4$ \\
\hline
\end{tabular}

Significantly different at p <0.05: ${ }^{a}$ vs. crystalline CCX; ${ }^{b}$ vs. $100 \%$ crystallized ASD; ${ }^{c}$ vs. $60 \%$ crystallized ASD.

*Data adapted from Knopp et al. (2016)

As can be seen in Figure 1 and Table 1, the in vitro $t_{\max }$ increased and the $\mathrm{AUC}_{0-4 \mathrm{~h}}$ and $C_{\max }$ decreased (almost linearly) with an increasing fraction of crystallized drug, respectively, which was somewhat expected given that both the dissolution rate and (apparent) solubility of the amorphous form of a drug is higher than that of its crystalline counterpart (Leuner and Dressman, 2000). This suggests that the performance of an ASD is significantly affected by crystallization of the drug in the ASD. By comparing the in vitro $C_{\max }$ for the crystalline CCX and the $100 \%$ crystallized ASD it is clear that PVP has some solubilizing properties, i.e. it 
increases the equilibrium solubility of crystalline CCX in the solution (from 54.9 to $60.8 \mu \mathrm{g} / \mathrm{mL}$ ) most likely due to drug-polymer interactions (Baghel et al., 2016). Therefore, based on the in vitro dissolution study it is expected that the mere presence of PVP in the $100 \%$ crystallized ASD will improve the performance of the system compared to pure crystalline CCX. Nevertheless, this improved performance pales in comparison with the effect from formulating the drug as an $\mathrm{ASD}$, which more than quadruples the in vitro $\mathrm{AUC}_{0-4 \mathrm{~h}}$.

This substantial increase in performance (AUC) of the pure ASD was also reflected in the in vivo study as shown in Figure 2 and Table 2. In accordance with the observations in vitro, the $t_{\max }$ for the pure and crystallized ASDs were significantly lower than for crystalline CCX, which is probably a result of the lower dissolution rate of the crystalline CCX compared to amorphous CCX. Similarly, both the in vivo $C_{\max }$ and $\mathrm{AUC}_{0-24 \mathrm{~h}}$ decreased with increasing fraction of crystallization with the pure ASD and the $20 \%$ and $40 \%$ crystallized ASDs being significantly higher than for the $100 \%$ crystallized ASDs (and crystalline CCX). Compared to 100\% crystallized ASD and crystalline CCX, the in vivo $\mathrm{AUC}_{0-24 \mathrm{~h}}$ for the pure ASD was approximately 2-fold and 3-fold higher, respectively, which indicates that crystallization has a significant impact on the in vivo performance of an ASD. In order to illustrate this influence, the in vivo $\mathrm{AUC}_{0-24 \mathrm{~h}}$ is plotted as a function of crystallized CCX fraction in Figure 3.

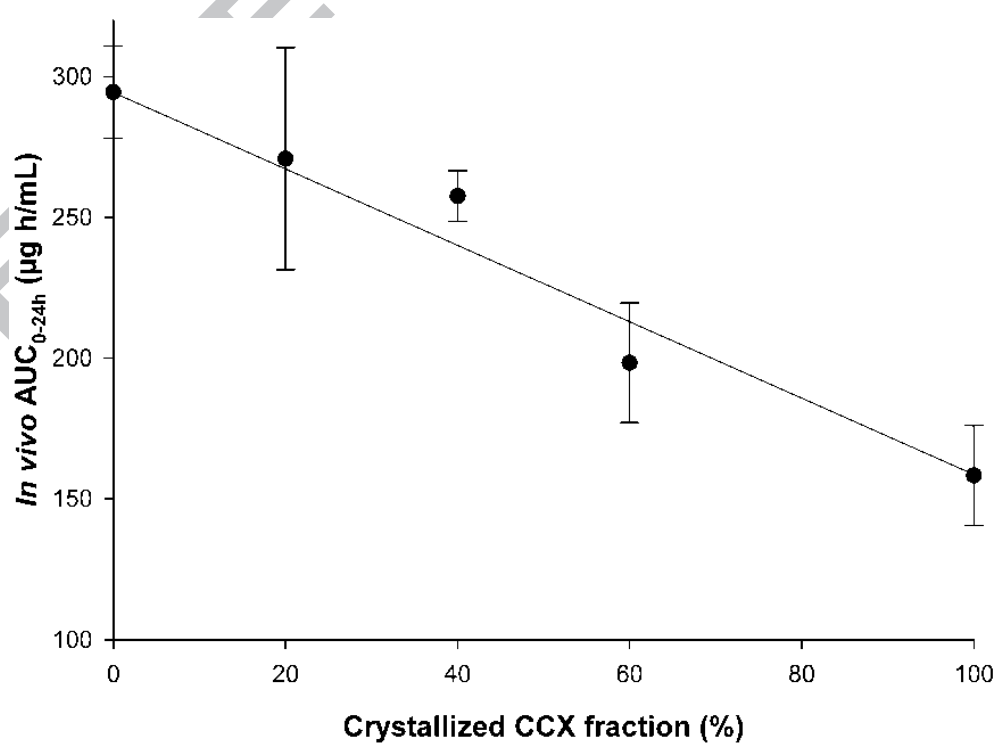

Figure 3: Relationship between the fraction of crystallized CCX (\% w/w) in the ASDs and in vivo $\mathrm{AUC}_{0-24 \mathrm{~h}} \pm \operatorname{SEM}(n=5)$.-The solid line in the figure is intended to indicate a linear 
relationship between the data points. The decrease in $\mathrm{AUC}_{0-24 \mathrm{~h}}$ as a function of crystallized CCX is fitted to a linear regression model illustrated by the solid line $\left(\mathrm{R}^{2}=0.961\right)$.

As can be seen, there is a good linear correlation between the in vivo $\mathrm{AUC}_{0-24 \mathrm{~h}}$ and fraction of crystallized CCX, which suggests that crystallization of a drug in an ASD during storage has significant impact on the in vivo performance or bioavailability of the drug. Nevertheless, the difference between the in vivo $\mathrm{AUC}_{0-24 \mathrm{~h}}$ and $C_{\max }$ for the pure $\mathrm{ASD}$ and the $20 \%$ and $40 \%$ crystallized ASDs was not statistically significant. This indicates that, despite the good correlation between the in vivo $\mathrm{AUC}_{0-24 \mathrm{~h}}$ and \% of crystallization of $\mathrm{CCX}$, these systems could be bioequivalent.

A test product can be considered bioequivalent with a reference product if the $90 \%$ confidence interval for AUC and $C_{\max }$ of the test product both fall within 80-125\% of those of the reference product (FDA, 2003). The $90 \%$ confidence interval for the test product is given by $X \pm Z \cdot s / V_{n}$, where $X$ is the mean, $Z$ is the standard normal variable ( $=1.645$ for $90 \%$ confidence interval), $s$ is the standard variation and $n$ is the number of samples. Hence, in order to be considered bioequivalent with the pure $\mathrm{ASD}$, the $90 \%$ confidence intervals for in vivo $\mathrm{AUC}_{0-24 \mathrm{~h}}$ and $C_{\max }$ for the crystallized ASDs should fall within 235.6-368.1 $\mu \mathrm{g} \cdot \mathrm{h} / \mathrm{mL}$ and $20.6-32.2 \mu \mathrm{g} / \mathrm{mL}$, respectively. For the $20 \%$ and $40 \%$ crystallized ASDs the $90 \%$ confidence intervals for in vivo $\mathrm{AUC}_{0-24 \mathrm{~h}}$ and $C_{\max }$ was 205.1-336.5 and 242.7-272.3 $\mu \mathrm{g} \cdot \mathrm{h} / \mathrm{mL}$, and 21.8-28.9 and 20.3-24.1 $\mu \mathrm{g} / \mathrm{mL}$, respectively. This means that the $20 \%$ crystallized ASD is bioequivalent to the pure ASD with regards to in vivo $C_{\max }$ and the $40 \%$ crystallized ASD was bioequivalent with regards to in vivo $\mathrm{AUC}_{0-24 \mathrm{~h}}$. However, as none of the crystallized ASDs fall within the limits of the pure ASD for both pharmacokinetic parameters, they cannot be considered bioequivalent.

The lack of bioequivalence between the pure ASD and the $20 \%$ crystallized ASD was probably a result of the high standard deviation on the $\mathrm{AUC}_{0-24 \mathrm{~h}}$ due to the relatively low number of animals $(n=5)$. Accordingly, bioequivalence between the $20 \%$ crystallized ASD and the pure ASD could probably be established if the number of animals is increased to $n=10-12$. In combination with the lack of statistical significance between the $\mathrm{AUC}_{0-24 \mathrm{~h}}$ and $C_{\max }$ for the pure ASD and the $20 \%$ and $40 \%$ crystallized ASDs, this could indicate that a small fraction (up to $20 \% \mathrm{w} / \mathrm{w}$ ) of crystallized drug in an ASD only have limited or no impact on the in vivo performance and bioavailability. In fact, considering that the crystals formed within an ASD during storage were 
most likely much smaller than the micronized drug particles used in this study (nm vs. $\mu \mathrm{m}$ scale), the effect of crystallization on the performance of the ASD was expected to be even less pronounced than demonstrated above. Nevertheless, this claim would have to be investigated in further detail in future studies in humans.

\section{Amorphous phase separation}

The in vitro dissolution profiles and in vivo plasma concentration-time profiles following administration of the different amorphous phase-separated ASDs are shown in Figures 4 and 5 , respectively. The corresponding (pharmacokinetic) parameters, maximum (plasma) concentration $\left(C_{\max }\right)$ of $\mathrm{CCX}$, time to reach $C_{\max }\left(t_{\max }\right)$, and area under the dissolution $\left(\mathrm{AUC}_{0-4 \mathrm{~h}}\right)$ and plasma concentration-time curves $\left(\mathrm{AUC}_{0-24 \mathrm{~h}}\right)$ are provided in Tables 3 and 4, respectively.

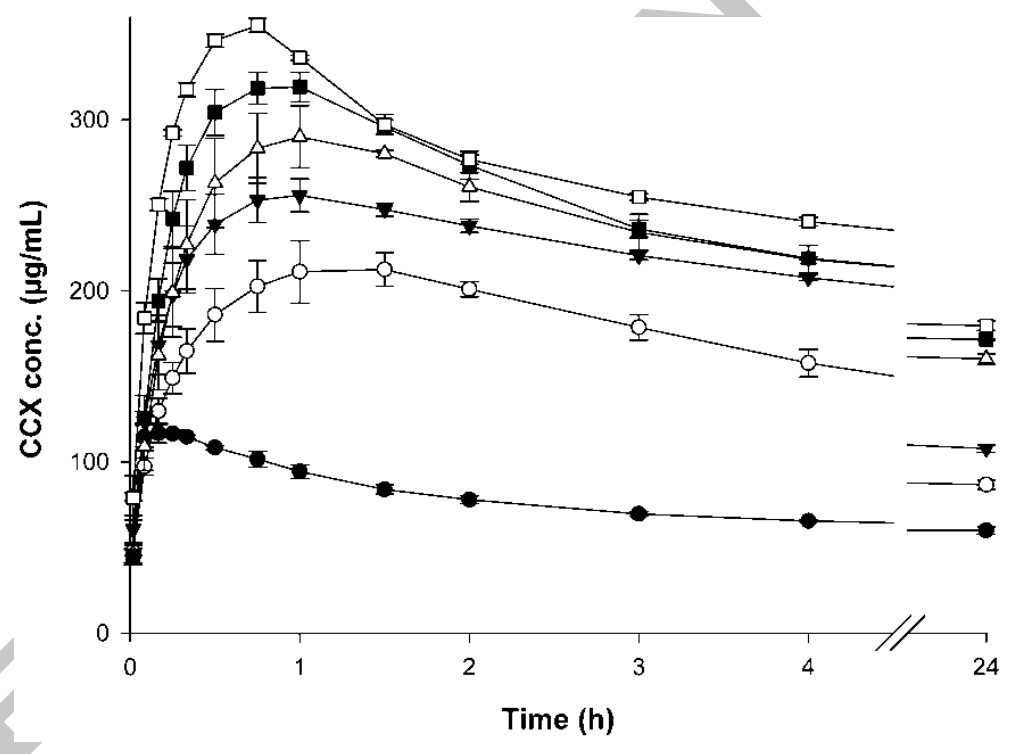

Figure 4: Dissolution profiles after non-sink in vitro dissolution of amorphous CCX (•), 100\% amorphous phase-separated ASD (०), 60\% amorphous phase-separated ASD ( $\mathbf{\nabla}), 40 \%$ amorphous phase-separated ASD $(\triangle), 20 \%$ amorphous phase-separated ASD $(\boldsymbol{\square})$ and pure ASD () at a dose of $400 \mathrm{mg}$ of CCX in $500 \mathrm{~mL}$ FaSSIF. Values represent mean CCX concentration \pm $\operatorname{SD}(n=3)$. 


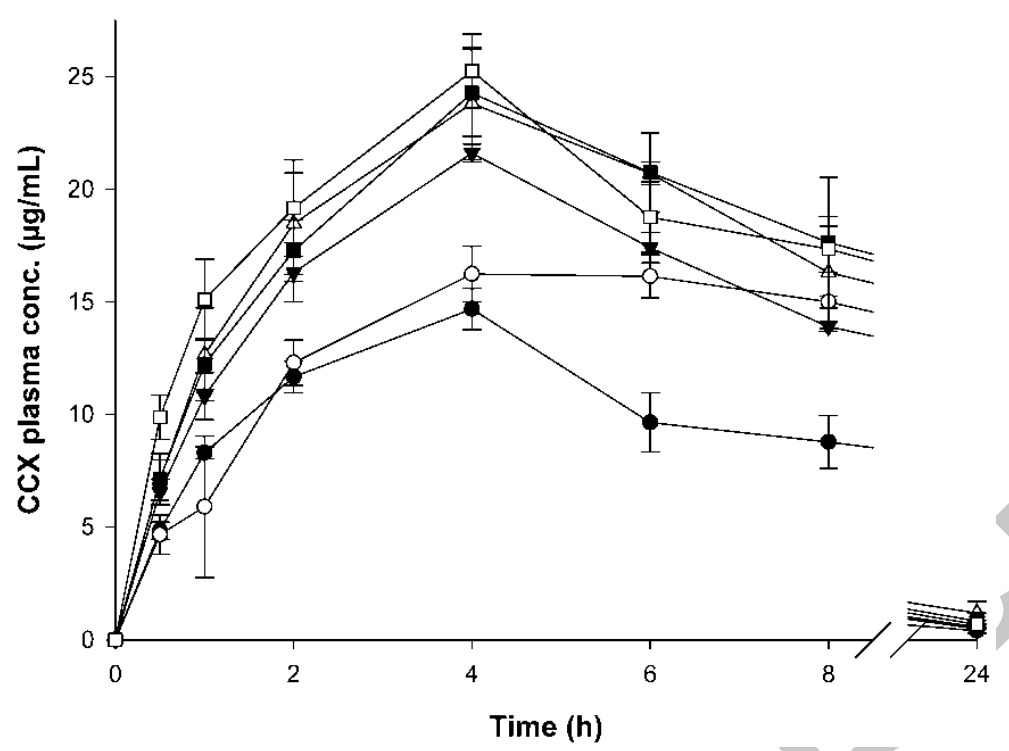

Figure 5: Plasma concentration-time profiles after oral administrations of amorphous CCX (•), $100 \%$ amorphous phase-separated ASD (०), 60\% amorphous phase-separated ASD ( $\mathbf{\nabla}), 40 \%$ amorphous phase-separated ASD $(\triangle), 20 \%$ amorphous phase-separated ASD (匹) and pure ASD ( $\square$ ) in rats at a dose corresponding to $100 \mathrm{mg} / \mathrm{kg}$ body weight of CCX. Values represent mean plasma concentration $\pm \operatorname{SEM}(n=3-5)$.

Table 3: In vitro parameters after non-sink dissolution testing of the amorphous phaseseparated ASDs at a dose of $400 \mathrm{mg}$ (values are mean $\pm \mathrm{SD}, n=3$ ).

\begin{tabular}{|l|l|l|l|}
\hline Formulation & $\mathbf{A U C}_{\mathbf{0 - 4}} \mathbf{~}\left(\mathbf{m g} \mathbf{~ m i n} \mathbf{~ m L}^{-\mathbf{1}}\right)$ & $\boldsymbol{C}_{\max }\left(\boldsymbol{\mu g} \mathbf{~ m L}^{-\mathbf{1}}\right)$ & $\boldsymbol{t}_{\max }(\mathbf{m i n})$ \\
\hline Pure ASD & $67.2 \pm 0.3$ & $355.2 \pm 4.0$ & $45.0 \pm 0.0$ \\
\hline $\begin{array}{l}\mathbf{2 0 \%} \text { amorphous } \\
\text { phase-separated ASD }\end{array}$ & $62.6 \pm 1.6$ & $320.0 \pm 9.7$ & $55.0 \pm 8.7$ \\
\hline $\begin{array}{l}\mathbf{4 0 \%} \text { amorphous } \\
\text { phase-separated ASD }\end{array}$ & $58.9 \pm 1.3$ & $292.7 \pm 13.9$ & $70.0 \pm 17.3$ \\
\hline $\begin{array}{l}\mathbf{6 0 \%} \text { amorphous } \\
\text { phase-separated ASD }\end{array}$ & $54.3 \pm 1.4$ & $256.4 \pm 10.3$ & $55.0 \pm 8.7$ \\
\hline $\begin{array}{l}\mathbf{1 0 0 \%} \text { amorphous } \\
\text { phase-separated ASD }\end{array}$ & $44.1 \pm 0.9$ & $215.7 \pm 14.9$ & $80.0 \pm 17.3$ \\
\hline Amorphous CCX & $19.8 \pm 0.3$ & $118.2 \pm 4.7$ & $13.3 \pm 2.9$ \\
\hline
\end{tabular}

Table 4: Pharmacokinetic parameters of the various amorphous phase-separated ASDs administered to rats at a dose of $100 \mathrm{mg} / \mathrm{kg}$ (values are mean $\pm \mathrm{SEM}, \boldsymbol{n = 3 - 5}$ ). 


\begin{tabular}{|c|c|c|c|}
\hline Formulation & $\operatorname{AUC}_{0-24 h}\left(\mu g h \mathrm{~mL}^{-1}\right)$ & $C_{\max }\left(\mu \mathrm{g} \mathrm{mL} \mathrm{L}^{-1}\right)$ & $t_{\max }(\mathrm{h})$ \\
\hline Pure ASD & $294.4 \pm 16.4^{\mathrm{a}}$ & $25.7 \pm 1.4^{\mathrm{a}, \mathrm{b}}$ & $3.6 \pm 0.4$ \\
\hline $\begin{array}{l}20 \% \text { amorphous } \\
\text { phase-separated ASD }\end{array}$ & $294.0 \pm 35.4^{\mathrm{a}}$ & $24.3 \pm 1.9^{\mathrm{a}, \mathrm{b}}$ & $4.0 \pm 0.0$ \\
\hline $\begin{array}{l}40 \% \text { amorphous } \\
\text { phase-separated ASD }\end{array}$ & $285.9 \pm 18.0^{\mathrm{a}}$ & $24.6 \pm 1.7^{\mathrm{a}, \mathrm{b}}$ & $5.3 \pm 1.3$ \\
\hline $\begin{array}{l}60 \% \text { amorphous } \\
\text { phase-separated ASD }\end{array}$ & $242.9 \pm 5.2^{\mathrm{a}}$ & $21.6 \pm 0.4^{\mathrm{a}, \mathrm{b}}$ & $4.0 \pm 0.0$ \\
\hline $\begin{array}{l}100 \% \text { amorphous } \\
\text { phase-separated ASD }\end{array}$ & $231.0 \pm 4.3^{\mathrm{a}}$ & $16.5 \pm 1.0$ & $5.3 \pm 1.3$ \\
\hline Amorphous CCX & $157.0 \pm 14.9$ & $14.9 \pm 0.8$ & $3.6 \pm 0.4$ \\
\hline
\end{tabular}

Significantly different at $\mathrm{p}<0.05:{ }^{\mathrm{a}}$ vs. amorphous CCX; ${ }^{\mathrm{b}}$ vs. $100 \%$ amorphous phase-separated ASD

As can be seen in Figure 4 and Table 3, the in vitro $t_{\max }$ generally decreased-increased with increasing fraction of amorphous phase-separated CCX (except for the 60\% phase-separated ASD). However, the $t_{\max }$ for the pure amorphous CCX was shorter than for the pure ASD (13.3 vs. $45.0 \mathrm{~min}$ ). This difference could be due to the dissolution rate of the amorphous CCX being higher than the dissolution rate of PVP as drug release from an ASD is driven by the dissolution of the polymer (Newman et al., 2012). However, by comparing the dissolution profile of the amorphous CCX with the $100 \%$ amorphous phase-separated ASD it is clear that PVP, besides having solubilizing properties (as shown in Table 1), also inhibited the crystallization of the drug from the supersaturated solution by delaying the onset of crystallization as implied from the increase in $t_{\max }$. This increase in dissolution rate followed by inhibition/delay of crystallization of drug from the supersaturated solution is a well-known property of many polymeric ASDs (such as CCX-PVP) and based on the appearance of the non-sink dissolution profile it is referred to as the "spring and parachute" effect (Baghel et al., 2016; Guzman et al., 2007). Furthermore, the in vitro $\mathrm{AUC}_{0-4 \mathrm{~h}}$ and $C_{\max }$ decreased with increasing fraction of amorphous phase separation, however, not to the same extent as for the crystallized ASDs (Figure 1 and Table 1). In fact, the difference between the in vitro $\mathrm{AUC}_{0-4 \mathrm{~h}}$ for the pure $\mathrm{ASD}$ and the $20 \%$ and $40 \%$ amorphous phase-separated ASDs was only around 10\%, which suggests that based on the in vitro dissolution study, amorphous phase separation may not have a significant influence on the performance of an ASD. These findings are in accordance with a study by Chen et al. (2018), who demonstrated that amorphous phase separation of a slow crystallizing drug in an ASD only had minimal impact on the in vitro dissolution performance. 
Indeed, at first glance, amorphous phase separation did not seem to have significant influence on the in vivo performance (in vivo $\mathrm{AUC}_{0-4 \mathrm{~h}}$ and $C_{\max }$ ) of the $\mathrm{ASD}$ as shown in Figure 5 and Table 4. In contrast to the in vivo study of the crystallized ASDs, there was no significant difference between $t_{\max }$ of the pure and amorphous phase-separated ASDs and amorphous CCX. However, in accordance with the observations in vitro (despite a lack of statistical significance), the in vivo $t_{\max }$ of for amorphous CCX was lower than that for all the crystallized ASDs, which was probably a result of the absence of the precipitation inhibitor PVP. In addition, both the in vivo $C_{\max }$ and $\mathrm{AUC}_{0-24 \mathrm{~h}}$ decreased with increasing fraction of crystallization with both pharmacokinetic parameters for the pure ASD and the 20\% and 40\% amorphous phase-separated ASDs being significantly higher than $100 \%$ phase-separated ASD and amorphous CCX. Nevertheless, compared to $100 \%$ phase-separated ASD and amorphous CCX, the in vivo $\mathrm{AUC}_{0 \text { - }}$ 24h for the pure ASD was only 1.3-fold and 1.9-fold higher, respectively, which suggest that the influence of phase separation of a drug in an ASD, may not be as significant, at least when compared to the effect crystallization. In order to illustrate the influence of amorphous phase separation on the in vivo performance of an $\mathrm{ASD}$, the in vivo $\mathrm{AUC}_{0-24 \mathrm{~h}}$ is plotted as a function of amorphous phase-separated CCX fraction in Figure 6.

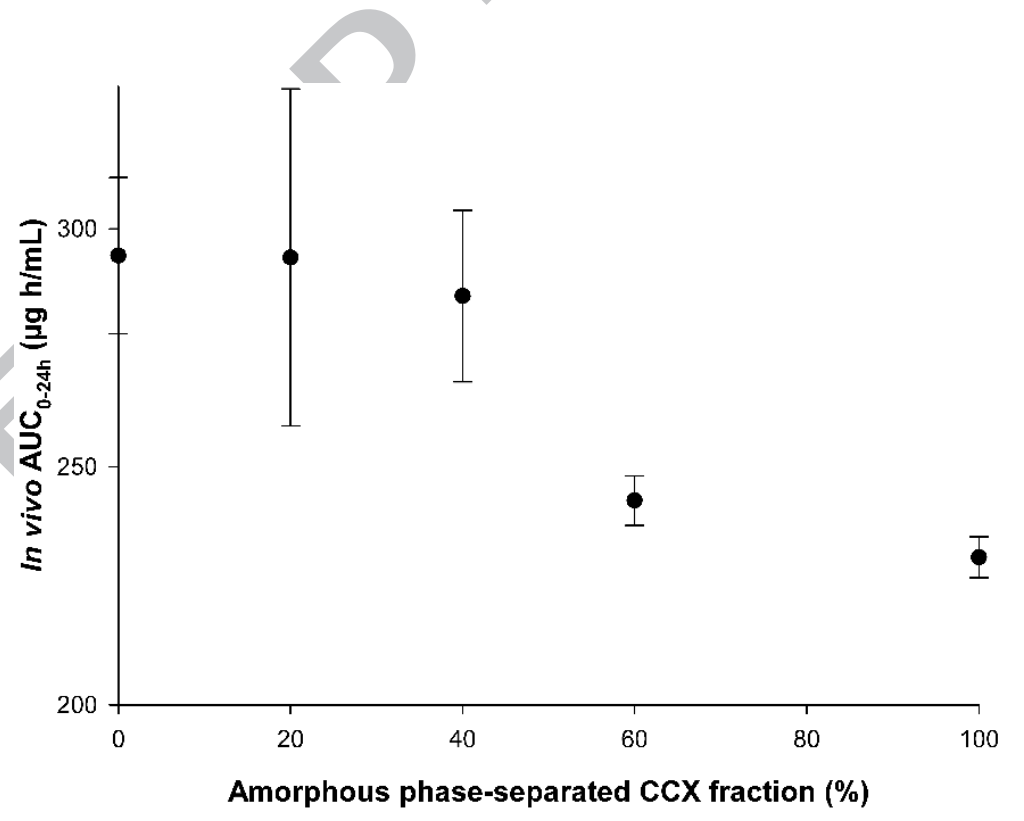

Figure 6: Relationship between the fraction of amorphous phase-separated CCX (\% w/w) in the $\mathrm{ASDs}$ and in vivo $\mathrm{AUC}_{0-24 \mathrm{~h}} \pm \mathrm{SEM}(n=3-5)$. The solid line in the figure is not intended to indicate a linear relationship between the data points. 
As can be seen, in contrast to the data presented in Figure 3, the in vivo $\mathrm{AUC}_{0-24 \mathrm{~h}}$ does not appear to decrease linearly with increasing fraction of amorphous phase-separated CCX. Rather it seems that the curve is S-shaped, which indicates that lower fractions of amorphous phase separation $(\leq 40 \% \mathrm{w} / \mathrm{w})$ do not affect the performance of an ASD. In fact, the lack of statistical significant differences between the $\mathrm{AUC}_{0-24 \mathrm{~h}}$ and $C_{\max }$ for the pure ASD and the amorphous phase-separated ASDs suggests that the these systems are bioequivalent.

As mentioned above, in order to be bioequivalent with the pure ASD, the $90 \%$ confidence intervals should fall within 235.6-368.1 $\mu \mathrm{g} \cdot \mathrm{h} / \mathrm{mL}$ for $\mathrm{AUC}_{0-24 \mathrm{~h}}$ and $20.6-32.2 \mu \mathrm{g} / \mathrm{mL}$ for $C_{\max }$. For the $20 \%$ and $40 \%$ amorphous phase-separated ASDs, the $90 \%$ confidence intervals for AUC $_{0-24 \mathrm{~h}}$ is $235.8-352.1$ and $256 .-315.5 \mu \mathrm{g} \cdot \mathrm{h} / \mathrm{mL}$, and the $90 \%$ confidence intervals for $C_{\max }$ is 21.1-27.4 and 21.7-27.4 $\mu \mathrm{g} / \mathrm{mL}$, respectively. This means that the $20 \%$ and $40 \%$ amorphous phase-separated ASDs can indeed be considered bioequivalent with the pure ASD. In combination with the lack of statistical significance between the $\mathrm{AUC}_{0-24 \mathrm{~h}}$ and $C_{\max }$ for the pure ASD and these systems, this strongly indicates that even a substantial fraction (up to $40 \% \mathrm{w} / \mathrm{w}$ ) of amorphous phase separation of the drug in an ASD during storage may not significantly impede the performance or bioavailability of the formulation.

\section{Conclusions}

The present study addresses the issues related to the efficiency and safety of crystallized or amorphous phase-separated ASDs as a result of the change in physicochemical properties of the system. The results demonstrate that both the in vitro and in vivo performance of an ASD was indeed influenced by crystallization or phase-separation of the drug. As expected, crystallization of CCX in the ASDs generally had a negative influence on performance compared to the pure ASD. However, the difference between the in vivo AUC of the pure ASD and the 20\% and 40\% crystallized ASDs was not statistically significant, which could indicate that a small fraction of crystallization of the drug in an ASD (e.g. below $20 \% \mathrm{w} / \mathrm{w}$ ) could only have limited or no impact on in vivo performance/ bioavailability. In comparison, amorphous phase separation of CCX in the ASDs did not negatively influence the in vitro and in vivo AUC to the same degree as crystallization. In fact, even though a slight decrease of in vivo AUC with increasing fraction of amorphous phase separation was observed, the $20 \%$ and $40 \%$ amorphous phase-separated ASDs were bioequivalent with the pure ASD. Even though only one drug-polymer systems was 
investigated, the findings of this study indicate that the eurrent regulatory-general reservations towards the impact of amorphous phase separation and crystallization of the drug in an ASD on its bioavailability may be exaggerated.

\section{References}

Abu-Diak, O., Jones, D.S., Andrews, G.P., 2011, An investigation into the dissolution properties of celecoxib melt extrudates: understanding the role of polymer type and concentration in stabilizing supersaturated drug concentrations. Mol Pharm, 8(4):1362-71.

Alonzo, D.E., Gao, Y., Zhou, D., Mo, H., Zhang, G. G.Z. and Taylor, L. S., 2011. Dissolution and precipitation behavior of amorphous solid dispersions. J. Pharm. Sci., 100, 3316-3331.

Andrews, G.P., Abu-Diak, O., Kusmanto, F., Hornsby, P., Hui, Z., Jones, D.S., 2010 Physicochemicalcharacterization and drug-release properties of celecoxib hot-melt extruded glass solutions. J Pharm Pharmacol, 62, 1580-90.

Augustijns, P. and Brewster, M. E., 2012, Supersaturating drug delivery systems: Fast is not necessarily good enough. J. Pharm. Sci., 101, 7-9.

Baghel, S., Cathcart, H., O'Reilly, N.J., 2016. Polymeric Amorphous Solid Dispersions: A Review of Amorphization, Crystallization, Stabilization, Solid-State Characterization, and Aqueous Solubilization of Biopharmaceutical Classification System Class II Drugs, J. Pharm. Sci., 105, 2527-2544.

Butler, J.M. and Dressman, J.B., 2010, The Developability Classification System: Application of Biopharmaceutics Concepts to Formulation Development, J. Pharm. Sci., 99, 4940 - 4954

Chen, H., Pui, Y., Liu, C., Chen, Z., Su, C.C., Hageman, M., Hussain, M., Haskell, R., Stefanski, K., Foster, K., Gudmundsson, O., Qian, F., 2018, Moisture-induced amorphous phase separation of amorphous solid dispersions: molecular mechanism, microstructure, and its impact on dissolution performance, J. Pharm. Sci., 107(1), 317-326.

Chokshi, R.J., Hossein, Z., Sandhu, H.K., Shah, N.H. Malick, W.A., 2007, Improving the Dissolution Rate of Poorly Water Soluble Drug by Solid Dispersion and Solid Solution-Pros and Cons, Drug Del., 14, 33-45.F

Fahr, A. and Liu, X., 2007, Drug delivery strategies for poorly water-soluble drugs, Expert Opin. Drug Deliv. 4, 403-416. 
Gupta, P., Chawla, G., \& Bansal, A. K., 2004a, Physical stability and solubility advantage from amorphous celecoxib: the role of thermodynamic quantities and molecular mobility. Mol. Pharm., 1, 406-413.

Gupta, P., Kakumanu, V.K., Bansal, A.K., 2004b, Stability and solubility of celecoxib-PVP amorphous dispersions: A molecular perspective, Pharm Res., 21, 1762-9.

Gupta, P., Thilagavathi, R., Chakraborti, A.K., Bansal, A.K., 2005, Role of molecular interaction instability of celecoxib-PVP amorphous systems. Mol Pharm, 2, 384-91.

Guzman, H. R., Tawa, M., Zhang, Z., Ratanabanangkoon, P., Shaw, P., Gardner, C. R., Chen, H., Moreau, J. P., Almarsson, O., Remenar, J. F., 2007, Combined use of crystalline salt forms and precipitation inhibitors to improve oral absorption of celecoxib from solid oral formulations. J. Pharm. Sci., 96(10), 2686-2702.

Hancock, B. C. and Zografi, G., 1997. Characteristics and significance of the amorphous state in pharmaceutical systems. J. Pharm. Sci., 86, 1-12.

He, Y. and Ho, C., 2015, Amorphous Solid Dispersions: Utilization and Challenges in Drug Discovery and Development. J. Pharm. Sci., 104, 3237-3258.

Hoei, Y.; Yamaura, K.; Matsuzawa, S., 1992, A lattice treatment of crystalline solventamorphous polymer mixtures on melting point depression. J. Phys. Chem., 96, 10584-10586.

Huang, Y., Dai, W.-G., 2014, Fundamental aspects of solid dispersion technology for poorly soluble drugs, Acta Pharm Sin B, 4, 18-25.

Klein, S, 2010, The use of biorelevant dissolution media to forecast the in vivo performance of a drug AAPS J, 12, 397-406.

Knopp, M. M., Olesen, N. E., Holm, P., Löbmann, K., Holm, R., Langguth, P., Rades, T., 2015. Evaluation of drug-polymer solubility curves through formal statistical analysis: Comparison of preparation techniques. J. Pharm. Sci, 104(1), 44-51.

Knopp, M. M., Nguyen, J. H., Mu, H., Langguth, P., Rades, T., Holm, R., 2016. Influence of copolymer composition on in vitro and in vivo performance of celecoxib-PVP/VA amorphous solid dispersions. AAPS J., 18(2), 416-423.

Leuner, C. and Dressman, J., 2000. Improving drug solubility for oral delivery using solid dispersions, Eur. J. Pharm. Biopharm., 50, 47-60.

Marsac, P.J., Li, T., Taylor, L.S., 2009, Estimation of Drug-Polymer Miscibility and Solubility in Amorphous Solid Dispersions Using Experimentally Determined Interaction 
Parameters. Pharm Res, 26, 139-51.

Newman, A., Knipp, G. and Zografi, G., 2012. Assessing the performance of amorphous solid dispersions. J. Pharm. Sci., 101, 1355-1377.

Rahman, Z., Siddiqui, A., Gupta, A., \& Khan, M., 2014, Regulatory considerations in development of amorphous solid dispersions. In Amorphous Solid Dispersions (pp. 545-563). Springer, New York, NY.

Shono, Y., Jantratid, E., Janssen, N., Kesisoglou, F., Mao, Y., Vertzoni, M., et al., 2009, Prediction of food effects on the absorption of celecoxib based on biorelevant dissolution testing coupled with physiologically based pharmacokinetic modeling. Eur J Pharm Biopharm, 73, 10714.

Stegemann, S., Leveiller, F., Franchi, D., de Jong, H., Linden, H., 2007. When poor solubility becomes an issue: from early stage to proof of concept, Eur. J. Pharm. Sci., 31, 249-261

US Food and Drug Administration, 2003. Guidance for Industry: Bioavailability and Bioequivalence Studies for Orally Administered Drug Products-General Considerations.

Van den Mooter, G., 2012, The use of amorphous solid dispersions: A formulation strategy to overcome poor solubility and dissolution rate, Drug Discovery Today: Technologies, 9, 79-85. Van den Mooter, G., Wuyts, M., Blaton, N., Busson, R., Grobet, P., Augustijns, P., et al., 2000 Physical stabilisation of amorphous ketoconazole in solid dispersions with polyvinylpyrrolidone K25. Eur J Pharm Sci, 12, 261-9.

Vo, C.L.-N., Park, C., Lee, B.-J., 2013. Current trends and future perspectives of solid dispersions containing poorly water-soluble drugs, Eur. J. Pharm. Biopharm., 85, 799-813.

Warren, D.B., Benameur, H., Porter, C.J.H., Pouton, C.W., 2010, Using polymeric precipitation inhibitors to improve the absorption of poorly water-soluble drugs: A mechanistic basis for utility. J Drug Target, 18, 704-31.

Xie, T., Taylor, L.S., 2015, Dissolution Performance of High Drug Loading Celecoxib Amorphous Solid Dispersions Formulated with Polymer Combinations. Pharm Res. 2015;1-12.

Xu, S. and Dai, W.-G., 2013, Drug precipitation inhibitors in supersaturable formulations, Int. J. Pharm., 453, 36-43. 


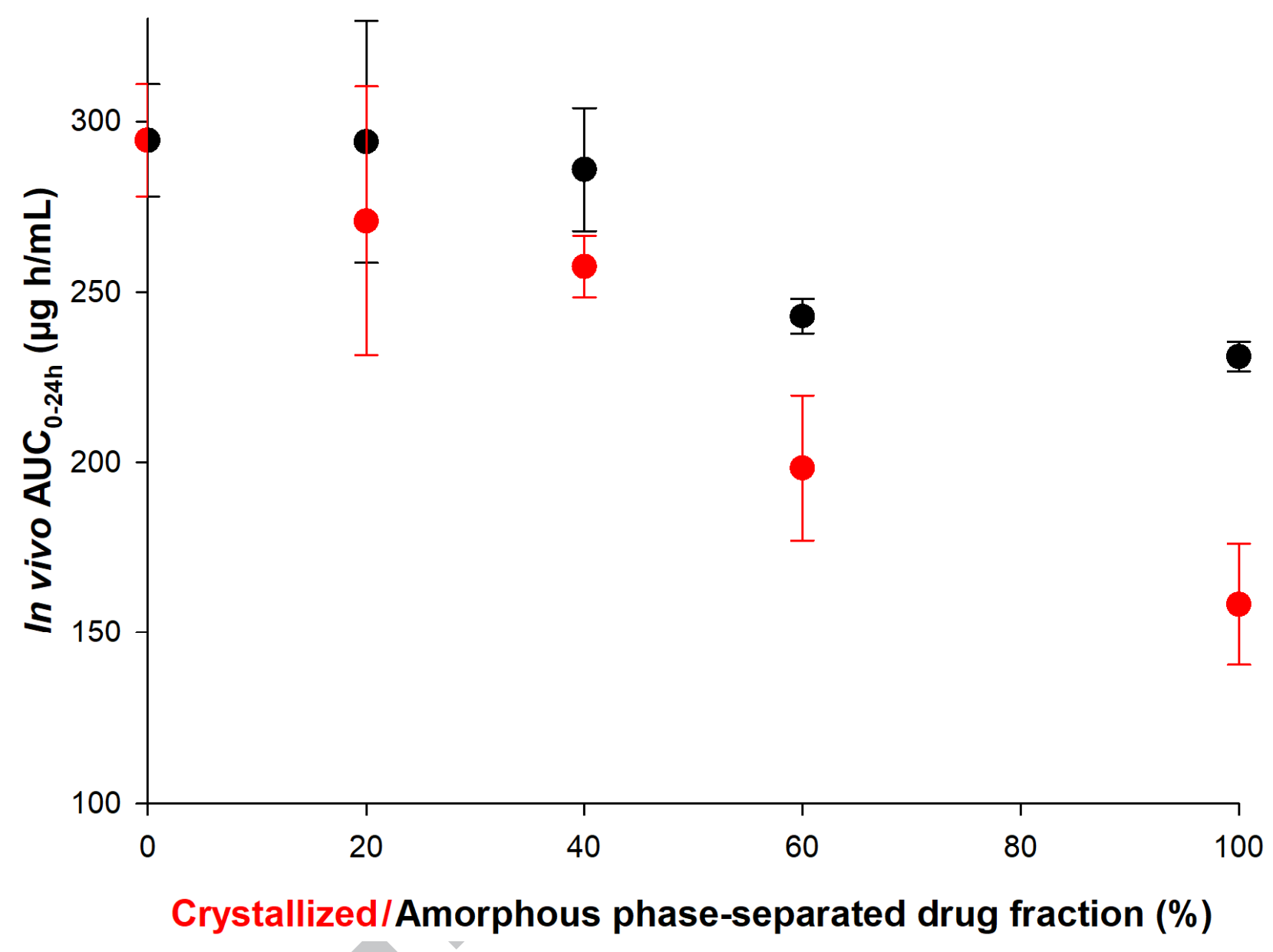

